\title{
Statistical analysis of yield trials by AMMI analysis of genotype $\times$ environment interaction
}

\section{Kuang Hongyu ${ }^{1,2}$, Marisol García-Peña ${ }^{1}$, Lúcio Borges de Araújo ${ }^{3}$, Carlos Tadeu dos Santos Dias ${ }^{4}$}

\footnotetext{
${ }^{1}$ Programa de Pos-graduação em Estatística e Experimentação Agronômica, Universidade de São Paulo, Brazil, e-mail: kuang.usp@gmail.com ${ }^{2}$ Departamento de Estatística, Universidade Federal de Mato Grosso, Av. Fernando Corrêa da Costa, no 2367 - Bairro Boa Esperança. 78060-900, Cuiabá - MT - Brazil

${ }^{3}$ Departamento de Estatística, Universidade Federal de Uberlândia, MG, Brazil

${ }^{4}$ Departamento de Ciências Exatas, Universidade de São Paulo, Brazil
}

\section{SUMMARY}

The genotype by environment interaction (GEI)) has an influence on the selection and recommendation of cultivars. The aim of this work is to study the effect of GEI and evaluate the adaptability and stability of productivity ( $\mathrm{kg} / \mathrm{ha}$ ) of nine maize genotypes using AMMI model (Additive Main effects and Multiplicative Interaction). The AMMI model is one of the most widely used statistical tools in the analysis of multiple-environment trials. It has two purposes, namely understanding complex GEI and increasing accuracy. Nevertheless, the AMMI model is a widely used tool for the analysis of multiple-environment trials, where the data are represented by a two-way table of GEI means. In the complete tables, least squares estimation for the AMMI model is equivalent to fitting an additive two-way ANOVA model for the main effects and applying a singular value decomposition to the interaction residuals. It assumes equal weights for all GEI means implicitly. The experiments were conducted in twenty environments, and the experimental design was a randomized complete block design with four repetitions. The AMMI model identified the best combinations of genotypes and environments with respect to the response variable. This paper concerns a basic and a common application of AMMI: yield-trial analysis without consideration of special structure or additional data for either genotypes or environments.

Key words: genotype environment interaction (GEI), adaptability and stability, additive main effects and multiplicative interaction model, multienvironment trials 


\section{Introduction}

Genotype by environment interaction (GEI) reflects the different responses of the genotypes to environment conditions, e.g., the best genotypes in an specific environment could not be the best for others. It depends on the considered environment. Therefore, GEI can-not represent all genetic potential environmental conditions, this makes difficult the recommendation of genotypes by the breeder (Gauch, 1992; Falconer and Mackay, 1996; ArciniegasAlarcn et al., 2011; Gauch et al., 2011; Gauch, 2013).

A differential response of genotypes across environments (often locationby-year combinations) is frequent in multiple-environment trials (METs) and is known as GEI (Rodrigues et al., 2014). MET data are often summarized in two-way tables, which have rows with the means of genotypes and columns with the environments. When the ranking of the genotypes changes across environments, the GEI occurs in various forms. In has the most extreme form it consists of crossover interactions. An example may be a genotype which is superior under wet conditions but may yield poorly under dry conditions (Rodrigues et al., 2014).

The additive main effects and multiplicative interaction (AMMI) model (Gauch, 1992) is one of the most widely used statistical methods. It can be used to understand and structure interactions between genotypes and environments. In its essence, the AMMI model applies the singular value decomposition (SVD) to the residuals of an additive two-way analysis of variance (ANOVA) model as applied to the GEI table of means (Gauch, 2013; Rodrigues et al., 2014). The two main purposes of AMMI analysis of a yield trial's treatment design are: (i) understanding complex GEI, which includes delineating mega-environments and selecting genotypes to exploit narrow adaptations; and (ii) increasing accuracy to improve recommendations, repeatability, selections, and genetic gains. The main purposes of an experimental design are assigning experimental units to treatments, quantifying errors, and gaining accuracy (Gauch, 2013).

In breeding, the researcher is interested in choosing the genotypes with superior performance in different environments. Poor efficiency in the genotype-by-environment interaction analysis of variance can represent a problem for breeders, who can take advantage of the interaction effect to choose genotypes with high productivity.

By accessing the GEI, many statistical methods are available to try understand better this effect. The choice of an appropriate statistical method 
depends on the experimental data, the number of available environments, and the accuracy of the information. Applications of the AMMI model to yield trials have appeared frequently during the last two decades, and there have been several recent review articles (Dias and Krzanowski, 2006; Gauch, 2006; Yan et al., 2007; Gauch et al., 2008; Yan et al., 2009; Gauch, 2013; Rodrigues et al., 2014). This analysis can help in the identification of genotypes which have high productivity and are well adapted to an agro-

nomic zone, with the aim of regionalised recommendation and selection of test sites (Gauch and Zobel, 1996; Gauch et al., 2011; Gauch, 2013).

This basic AMMI analysis is important due to its common nature characteristic and its basic case must be mastered as a foundation for any of the more complicated applications (Gauch, 2013). For instance, a preliminary AMMI analysis to gain accuracy can greatly improve subsequent analyses for generating hierarchical classifications (Smith and Gauch, 1992) or detecting quantitative trait loci (Gauch et al., 2011).

The objective of this work is to study the effect of GEI. By using the AMMI model, it assesses the adaptability and stability of productivity of nine maize genotypes in twenty environments.

\section{Material and methods}

The data used in this work are the same as in Cornelius and Crossa (1999). The above outline of basic AMMI analysis can be illustrated using an international trial for grain yield $(\mathrm{kg} / \mathrm{ha})$. The data were obtained by the International Maize and Wheat Improvement Center (CIMMYT) in trials conducted in 20 countries. This analysis used 9 genotypes of maize, and each genotype was assessed in 20 environments. The experiment had an RCB design with 4 replications, but only the averages over replications are available from the publication, as well as the mean square error.

The data for production $(\mathrm{kg} / \mathrm{ha})$ are analysed with individual ANOVA by location and by conjoint analysis. In order to assess the genetic variability between the treatments and the GEI, the data have to cover all the loctions. After detection of the presence of the interaction, an adaptability and phenotypic stability analysis were made using the AMMI model.

\subsection{Analysis of variance}

There are three numbers from the ANOVA which provide a preliminary indication as to whether AMMI analysis will be worthwhile. These are the 
sum of squares $(S S)$ for genotypes $(G)$, GEI signal $\left(G E_{S}\right)$, and GEI noise $\left(G E_{N}\right)$. The SS values for $\mathrm{G}$ and GEI are direct outputs from ANOVA (Gauch, 2013). To estimate the $S S$ for $G E_{N}$, simply multiply the error mean square (from replication) by the number of degrees of freedom $(d f)$ for GEI (Gauch, 1992). Then obtain $G E_{S}$ by subtracting $G E_{N}$ from GEI. AMMI analysis is appropriate for datasets that have substantial $G$ and substantial $G E_{S}$. Especially when the $S S$ for $G E_{S}$ is at least as large as that for $G$, as happens frequently, AMMI analysis will probably be worthwhile (Gauch, 1992; Gauch, 2013). On the other hand, occasionally GEI is buried in noise, with the SS for $G E_{N}$ approximately equal to that for GEI. In that case, GEI should be ignored, so AMMI analysis isinappropriate (Gauch, 2013).

\subsection{Model diagnosis}

AMMI is a model family rather than a single model. Consequently, model diagnosis is required to determine which member of this model family is the best for a given dataset and research purpose (Gauch, 2006; Gauch, 2013). The AMMI model combines two methods: analysis of variance and singular value decomposition in a unique model, additive components for the main effects of genotypes $\left(g_{i}\right)$, environments $\left(e_{j}\right)$ and multiplicative components for the interaction effect $(g e)_{i j}$ (Gauch, 1992; Dias and Krzanowski, 2003; García-Peña and Dias, 2009; Arciniegas-Alarcn et al., 2011; Gauch et al., 2011; Gauch, 2013).

Therefore, the model equation for the $i$-th genotype in the $j$-th environment in $r$ blocks (repetitions) is (Gauch, 1992):

$$
Y_{i j r}=\mu+g_{i}+e_{j}+b_{r}\left(e_{j}\right)+\sum_{k=1}^{n} \lambda_{k} \alpha_{i k} \gamma_{j k}+\rho_{i j}+\varepsilon_{i j}
$$

where $Y_{i j r}$ is the phenotypic trait (e.g. yield) of genotype $i$ in environment $j$ for replicate $r, \mu$ is the grand mean, $g_{i}$ are the genotype main effects as deviations from $\mu, e_{j}$ are the environment main effects as deviations from $\mu$, $\lambda_{k}$ is the singular value for the Interaction Principal Component (IPC) axis $k, \alpha_{i k}$ and $\gamma_{j k}$ are the genotype and environment IPC scores (i.e. the left and right singular vectors) for axis $k, b_{r}\left(e_{j}\right)$ is the effect of the block $r$ within the environment $j, r$ is the number of blocks, $\rho_{i j}$ is the residual containing all multiplicative terms not included in the model $(1) ; n$ is the number of axes or principal components (PC) retained by the model, and $\varepsilon_{i j r}$ is the experimental error, assumed independent with identical distribution, $\varepsilon_{i j} \sim N\left(0, \frac{\sigma^{2}}{r}\right)$. 
Successive IPCs are denoted by IPC1, IPC2, IPC3, and so on. The number of these components is one less than the minimum of the number of genotypes and number of environments. The member of the AMMI model family retaining zero components is denoted by AMMI0, and the successive members which retain one or more components are denoted by AMMI1, AMMI2, AMMI3, and so on, up to the full model retaining all components denoted by AMMIF (Gauch, 1992; Gauch, 2013). When the fitted values of the full model automatically equal the raw data $Y_{i j r}$ exactly, the residual term disappears. However, the reduced models leave a residual $\varepsilon_{i j r}$ (Gauch, 2013).

In the first phase of the main effects in the additive part (mean, genotypes and environments), they are fitted by a traditional AOV to the matrix of means $\left(\mathbf{Y}_{(g \times e)}\right)$ in the $r$ blocks. The result is the nonadditivity residual which means the GEI, $(g e)_{i j}$. This interaction is the multiplicative part of the model. In the second phase, the interaction is analysed by singular value decomposition $(\mathrm{SVD})$ of the matrix $\left(\mathbf{G E}_{(g \times e)}=\left[(g e)_{i j}\right]\right)$ or by principal component analysis (PCA) (Gauch, 1992; Dias and Krzanowski, 2003; Dias and Krzanowski, 2006; Gauch et al., 2011; Gauch, 2013).

The GE matrix is the interaction matrix between genotypes and environments (residual of main effects), where each element $(g e)_{i j}$ of $\mathbf{G E}$ is given by

$$
(g e)_{i j}=Y_{i j}-\bar{Y}_{i \bullet}-\bar{Y}_{\bullet j}+\bar{Y}_{\bullet \bullet}
$$

where $Y_{i j}$ is the mean of the repetitions of genotype $i$ in environment $j$, with $i=1,2, \ldots, g$ and $j=1,2, \ldots, e ; \bar{Y}_{i \bullet}$ is the mean of genotype $i, \bar{Y}_{\bullet} j$ is the mean of environment $j$ and $\bar{Y}$.• is the global mean.

There are many techniques to assign degrees of freedom in a AMMI model. One of them consists in determining the degrees of freedom associated with each part of the interaction sum of squares $S S_{G E I}$. It means, associated to $\lambda_{k}^{2}$. Each member of the AMMI model family is obtained from the mean square $(M S)$, and calculated by the $F$ test for each component to assess the significance in relation to the $M S_{\text {mean error }}$. The process results in variance analysis similar to the traditional one, unfolding the degrees of freedom corresponding to the GEI (Gauch, 1992).

In this way, number of axes to explain the behaviour of the interaction was determined by considering the proportion of the $S S_{G E I}$ accumulated up to the $n$-th axis $\left(\sum_{k=1}^{n} \lambda_{k}^{2} / S S_{G E I}\right)$ (Gauch, 1988). The stop point determines the model selected (AMMI0, AMMI1,..,AMMIF) based on the 
significance of the $F$ test for the successive terms of the interaction. The Gollob (1968) method is one of the most used to assign degrees of freedom to the AMMI model. The expression for the method is: $D F_{P C_{k}}=g+e-1-2 k$, with $k=1,2, \ldots, p, p=\min \{g-1, e-1\}$, where $P C_{k}$ is the $k$-th axis of the principal component.

Piepho (1995) showed that the $F_{R, n}$ test proposed by Cornelius et al. (1992) is more robust than that proposed by Gollob (1968); the statistic is defined as $F_{R, n}=\left(S S_{G E I}-\sum_{k-1}^{n} \lambda_{k}^{2}\right) /\left(f_{2} M S_{\text {mean error }}\right)$, where $f_{2}=$ $(g-1-n)(e-1-n)$ and $n$ is the number of multiplicative terms in the model. The $F_{R, n}$ statistic with null hypothesis that there are no more than $n$ terms determining the interaction. It has an approximate $F$ distribution with $f_{2}$ degrees of freedom and $D F_{\text {mean error }}$.

The results were analysed by graphical representation in biplot, a technique which is useful in principal component analysis. The graphic is used to represent simultaneously the rows and columns of a data matrix. It indicates the existing groups in the observations and in this way shows dispersions and correlations between the variables (Gauch, 1992).

The biplot was introduced by Gabriel (1971). It is based on the approximation of a matrix with rank 8 by the singular value decomposition (SVD), and by another matrix with lower rank. In the analysis of AMMI, the SVD is applied to the interaction matrix GE (matrix of rank $p$ ).

$$
\widehat{\mathbf{G E}}_{(g \times e)}=\sum_{k=1}^{n} \lambda_{k} \boldsymbol{\gamma}_{k} \boldsymbol{\alpha}_{k}^{\prime}=\underbrace{\widetilde{\mathbf{U}}}_{\left(\mathbf{P}_{B B^{\prime}}\right)} \underbrace{\widetilde{\mathbf{S}}}_{\left(\boldsymbol{\Lambda}^{1 / 2}\right)} \underbrace{\widetilde{\mathbf{V}}^{\prime}}_{\left(\mathbf{P}_{B^{\prime} B}\right)}
$$

where $\widetilde{\mathbf{U}}_{(g \times e)}$ has in its columns, the first $p$ vectors $\gamma_{(g \times 1)} ; \widetilde{\mathbf{S}}_{(n)}$ is a diagonal matrix with the first singular values $\lambda_{1}, \lambda_{2}, \ldots, \lambda_{p}$ with $n<p$ and $\mathbf{V}_{(g \times e)}$ has in its rows the first $n$ vectors $\boldsymbol{\alpha}_{(1 \times e)}^{\prime}$.

Therefore, analysis of AMMI gives the decomposition $\mathbf{G E}_{(g \times e)}=\mathbf{U S V}^{\prime}=$ $\mathbf{U S}^{1 / 2} \mathbf{S}^{1 / 2} \mathbf{V}^{\prime}=\mathbf{G H}^{\prime}$ and the approximate decomposition by $n$ components $\mathbf{G E}_{(g \times e)}=\widetilde{\mathbf{U}} \widetilde{\mathbf{S}} \widetilde{\mathbf{V}}^{\prime}=\widetilde{\mathbf{G}} \widetilde{\mathbf{H}}^{\prime}=\sum_{k=1}^{n}\left(\lambda_{k}^{1 / 2} \gamma_{k}\right)\left(\lambda_{k}^{1 / 2} \gamma_{k}^{\prime}\right)$ where $\mathbf{G}_{(g \times e)}$ is the genotype effect and $\mathbf{H}_{(n \times e)}$ the location effect(Gauch, 1992; Dias and Krzanowski, 2006; Gauch, 2006).

All the analyses were implemented in SAS (SAS, 2004).

\subsection{Mega-Environment Delineation}

When there are crossovers between winning genotypes, subdivision of a growing region into two or more mega-environments is necessary for exploit- 
ing the narrow adaptations that often provide a substantial opportunity to increase yield. Sometimes there are more than the broad adaptations. There are three important considerations (Gauch, 2013):

First, model diagnosis is essential because as the selected member of the AMMI model family changes, the mega-environments also change, with higher-order models tending to define a larger number of mega-environments. Consequently, mega-environments can-not be meaningfully or reliably delineated without first performing a model diagnosis to select the best member of the AMMI model family for a given dataset. Model diagnosis enables researchers to distinguish between $\mathrm{GE}_{S}$ causing actual narrow adaptations and $\mathrm{GE}_{N}$ generating spurious complexity (Gauch, 2013).

Second, it is important for mega-environments to have predictive potential for locations and years. This predictive role is greatly enhanced if the mega-environments, initially delineated in terms of winning genotypes, have an evidential and environmental interpretation in terms of environmental gradients or geographical subregions (Gauch, 2013). Predictable environmental factors associate with locations or management practices increase the number of usable mega-environments, whereas unpredictable environmental factors associated with years decrease the number and usefulness of mega-environments (Gauch, 1992; Annicchiarico, 1997; Annicchiarico, 2002; Gauch, 2013).

Third, because of the subdivision of several mega-environments, it is costly for breeding programmes and seed distributors alike unless a practical portion $\mathrm{GE}_{P}$ of the interaction signal $\mathrm{GE}_{S}$ becomes available for exploiting narrow adaptations to increase yields. This limitation makes it necessary to select a low-order model such as AMMI1 for delineating a small and manageable number of mega-environments, even though a higher model such as AMMI3 may be selected for the different research purpose of optimizing predictive accuracy (Gauch, 2013). But fortunately, just 2 or 3 mega-environments are often sufficient to allow $\mathrm{GE}_{P}$ to capture a sizable portion of $\mathrm{GE}_{S}$.

\subsection{Agricultural recommendations}

A major purpose of yield-trial research is to select the best genotypes to retain in a breeding programme or to recommend the best cultivars for a growing region. But the tasks of selection are remarkably difficult, due to the fact that their noise obscures true winners, generates spurious complexity, and reduces genetic gains (Gauch, 1992).A common approach for 
genotype selection pursues both high yield and stability, but this approach has five considerable problems (Gauch, 2013):

Firstly, there are dozens of stability parameters which make any particular choice difficult to justify, even though they can be organized into four groups (Annicchiarico, 2002; Gauch, 2013). Secondly, there are manifold ways to integrate high yield and stability, but many fail to optimize any known and agriculturally significant outcome. Thirdly, stability is a meaningful objective only within an individual mega-environment, not across multiple mega-environments, and yet much of the literature ignores this crucial requirement (Gauch, 2013). Fourthly, at least 8 trials within each mega-environment are necessary for reasonably reliable estimates of stability (Annicchiarico, 2002; Gauch, 2013). Yet researchers often use fewer trials. Fifthly and finally, regarding instability solely as a problem to be minimized is a defective paradigm because the complete story is that instability (GEI) presents plant breeders with both problems and opportunities (Gauch, 2013).

\section{Results and discussion}

The results of the joint analysis of variance for productivity of maize (kg/ha) show that the genotype, environment and interaction effects are significant $(p<0.01)$. That indicates that the genotypes present different behavior in the environments. It justifies a study of the behaviour of the genotypes in order to identify the magnitude of interaction with the environments (Gauch, 1992).

Table 1 presents the ANOVA for AMMI 8. The sources listed in this table correspond to the terms in Eq. (1) for the AMMI model applied to a yield trial with an RCB experimental design. In the AMMI analysis the square sum of the interaction $\left(S S_{G E I}=249704162\right)$ corresponds to the eigenvalues $\left(\sum_{k=1}^{n} \lambda_{k}^{2}\right)$ (Table 1$)$. It can be inflated by the presence of noise (inexplicable variation) in the response variable. It is necessary to make an adjustment of the interaction by singular value decomposition of the GEI matrix. The $\mathrm{SS}$ for $\mathrm{G}$ is 79828575 , for $\mathrm{GE}_{N}$ it is 91632744 , and for $\mathrm{GE}_{S}$ it is $249704162-91632744=158071418$ and for the irrelevant $\mathrm{E}$ it is 989593772 (Table 1). This matrix has rank $p=8$, therefore it can be decomposed into eight principal components that correspond to the partial square sum in the analysis of variance.

Model diagnosis for AMMI is routinely done using cross-validation of experiments with replication. However, this is not an option for this wheat 
Table 1. Conjoint analysis of variance of the productivity of maize trials and decomposition of the sum of squares of (GEI)

\begin{tabular}{|c|c|c|c|c|c|c|c|c|}
\hline Source & $\mathrm{DF}_{G}$ & SS & MS & $\mathrm{F}_{G}$ & $p$-value & $\mathrm{DF}_{C}$ & $\mathrm{~F}_{C}$ & $p$-value \\
\hline Environment (E) & 19 & 989593772 & 52083883 & 26.30 & $<0.01$ & - & - & - \\
\hline $\begin{array}{l}\text { Block/ } \\
\text { Environment }\end{array}$ & 60 & 118813054 & 1980218 & 3.28 & $<0.01$ & - & - & - \\
\hline Genotype (G) & 8 & 79828575 & 9978572 & 16.55 & $<0.01$ & - & - & - \\
\hline $\begin{array}{l}\text { Interaction } \\
(G \times E)\end{array}$ & 152 & 249704162 & 1642791 & 2.73 & $<0.01$ & 152 & 2.73 & $<0.01$ \\
\hline $\mathrm{PC} 1$ & 26 & 140323418 & 5397054.5 & 8.95 & $<0.01$ & 126 & 1.44 & $<0.01$ \\
\hline $\mathrm{PC} 2$ & 24 & 37711634 & 1571318.1 & 2.61 & $<0.01$ & 102 & 1.17 & 0.1487 \\
\hline PC3 & 22 & 26058572 & 1184480.6 & 1.96 & $<0.01$ & 80 & 0.9457 & 0.6116 \\
\hline $\mathrm{PC} 4$ & 20 & 21533133 & 1076656.9 & 1.79 & 0.0192 & 60 & 0.6657 & 0.9739 \\
\hline PC5 & 18 & 12371704 & 687316.9 & 1.14 & 0.3093 & 42 & 0.4623 & 0.9986 \\
\hline PC6 & 16 & 6900236 & 431264.7 & 0.72 & 0.7744 & 26 & 0.307 & 0.9997 \\
\hline $\mathrm{PC} 7$ & 14 & 2513071 & 179505.1 & 0.30 & 0.9939 & 0 & - & - \\
\hline PC8 & 12 & 2292394 & 191032.8 & 0.32 & 0.9858 & 0 & - & - \\
\hline Residual & 480 & 289366499 & 602847 & - & - & - & - & - \\
\hline Total & 719 & 1727306061 & - & - & - & - & - & - \\
\hline $\begin{array}{l}\text { General mean } \\
\mathrm{CV}(\%)\end{array}$ & $\begin{array}{r}1858.13 \\
15.982\end{array}$ & & & & & & & \\
\hline
\end{tabular}

trial because the replicated data were not published (Gauch, 2013). However, the mean square error has been published. This guides model diagnosis by retaining as many IPCs as needed for the sum of their eigenvalues to approximate the $\mathrm{SS}$ for $\mathrm{GE}_{S}$, namely 158071418. From Table 1, the AMMI2 model captures 178035052, which is close (and it is better not to add IPC3 with its SS of 26058572). Hence, the model diagnosis for optimizing predictive accuracy points to AMMI2.

Observing the decomposition of the genotype-by-environment interaction through the AMMI model (Table 1), the first, second and third principal components $(\mathrm{PCs})$ are significant $(p<0.01)$ by Gollob $(1968) F$ test, and explain $59.2 \%, 15.1 \%$ and $10.4 \%$ respectively of the variation of the $S S_{G E I}$. These three principal components sum to $81.7 \%$ of the $S S_{G E I}$, which is considered a pattern response present in the $S S_{G E I}$ with 72 degrees of freedom ( $47.37 \%$ of the interaction degrees of freedom).

Another alternative to choose the AMMI model is the $F_{R}$ test proposed by Cornelius, described by Piepho (1995) as one of the most robust. AMMI1 was chosen by the criteria model, with a $1 \%$ level of significance. This axis explained $56.2 \%$ of the $S S_{G E I}$, while the other axes represent variations of the $S S_{G E I}$ with a large amount of noise. In this way the model AMMI1 was the best to describe the pattern of the interaction (Table 1).

The results of unfolding the GEI correspond to each member of the AMMI model family, it means the AMMI0 model, all the GEI, with 152 
DF. After removing the $26 \mathrm{DF}$ and the sum of squares assigned to the first axis PC1, the AMMI1 model is the remainder of the interaction. After removing the $24 \mathrm{DF}$ and the sum of squares assigned to the second axis PC2, AMMI2 is the remainder and so on for the AMMI3 up to the AMMI8 model (Table 1).

The last stage of the AMMI analysis is the graphical representation of genotypes and environments in the biplot (Gabriel, 1971) and identification of mega-environment. It is necessary to determine the position in the interaction of singular axes. From the matrix $\mathbf{U}, \mathbf{S}$ and $\mathbf{V}$ resulting from the singular value decomposition (SVD) of the GE matrix, we obtain the results of interest (García-Peña and Dias, 2009).

In this case, it is possible to build up the biplot. The biplots are: i) AMMI1 - Means vs PC1 (Fig. 1); ii) AMMI2 - PC1 vs PC2 (Fig. 2). The biplot graphics are used to analyse the dispersion of genotypes, environments and the interaction between them. The AMMI1 biplot contains the variation of the principal additive effects of genotypes and environments. This is shown in the horizontal axis, while the variation of the multiplicative effects of the GEI is shown in the vertical axis. In the biplot, AMMI2 is visualized by the multiplicative effects of the GEI contained in the first two PCs.

The first singular axis of the AMMI analysis captures the highest percentage of the "pattern" of the data (Gauch, 1988). A high percentage of the $S S_{G E I}$ is explained by the first two axes $(71.2 \%)$, and the highest part of the "pattern" of the GEI will be captured. In this way, the scores of the genotypes and environments are plotted until the second axis corresponding to each variable.

According to the values of the two first principal components (CP1 and CP2) or by Fig. 1, G6, G5 G4 and G9 are the genotypes with best answers and more productive in the environmental conditions prevailing during crop development. The genotypes are more stable in G7, G2, G3 and G6 (Fig. 1 and 2). These can be grown in all the localtions where the study was carried out. Among them, the genotypes G6, G3 and G7 display a productivity above the general mean and are between the two groups (productive and stable). They indicate that these genotypes are associated with adaptability and stability. However, the genotypes with high mean productivity can-not be stable. The case of the genotype G5 shows a specific adaptability to the environment E15 and the genotype G4 to the environment E11 (Fig. 1 and Fig. 2). 


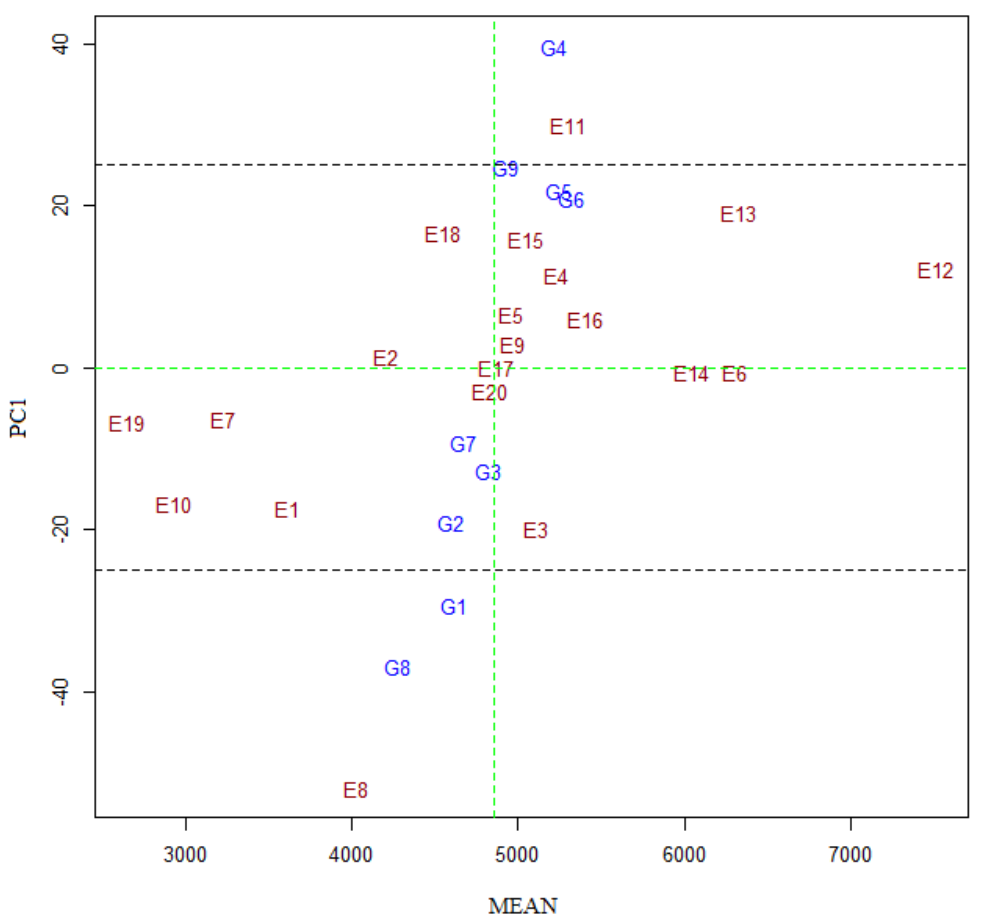

Figure 1. Biplot AMMI1 (Means vs PC1) for the data of productivity of maize $(\mathrm{kg} / \mathrm{ha})$ with nine genotypes $(\mathrm{G})$ and twenty environments $(\mathrm{E})$

To determine if mega-environments can be observed, we consider Fig. 2. Five mega-environments are observed, only two of them being considered of importance. The first, called mega-environment A, is considered the most appropriate, since it has the most productive and stable genotypes, also the environments where these materials exhibit better behaviour. This megaenvironment is formed by genotypes G6 and G5. The second best, called mega-environment B, is formed by genotypes G4 and G9.

The specific adaptation indicates a high mean productivity of a genotype in a selected environment. For example, genotype G4 and G9 are adapted to the environments E11, E13 and E18, and the genotypes G5 and G6 are adapted to the environment E15, etc. (Fig. 2).

As regards the environments, poor contributions were presented by the environments E9, E16, E14, E4, E5, E19 and E7 (Fig. 2). The environments more discrepant were E19, E10, E8, E13 and E11. E12 gives the highest mean of productivity $(7516.500 \mathrm{~kg} / \mathrm{ha})$ and E19 the lowest mean $(4837.614$ 


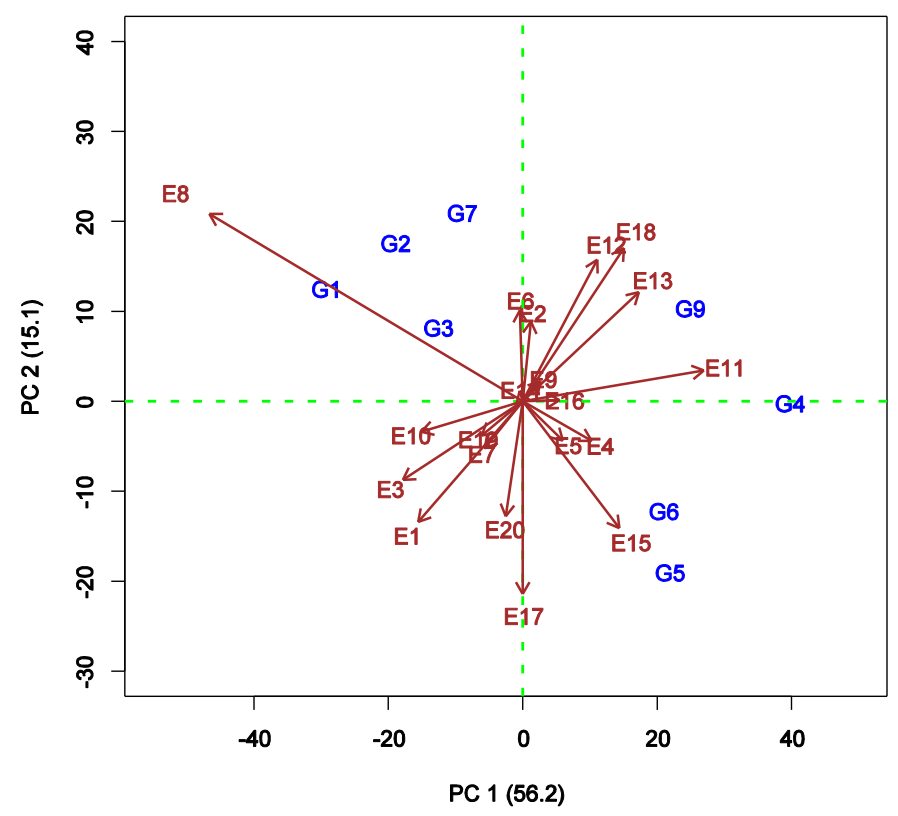

Figure 2. AMMI2 biplot (PC1 vs PC2) for data on the productivity of maize $(\mathrm{kg} / \mathrm{ha})$ with nine genotypes $(\mathrm{G})$ and twenty environments $(\mathrm{E})$

$\mathrm{kg} / \mathrm{ha}$ ). These can be considered examples of favourable and unfavourable environments, respectively (Fig. 1 and 2). The environments E20 and E17, E9 and E5 are very similar. This information can be observed in Fig. 1. It is possible to replace one of this locals by other more representative of the region, where the cultivars will be recommended. The AMMI analysis can be used efficiently in the identification of superior environmental conditions for agricultural exploitation (selection of growing locations) and superior mean performance genotypes (Gauch et al., 2008; Yan, 2010).

\section{Conclusions}

The genotypes G6, G3 and G7 display higher adaptability and stability. Therefore they are recommended to be used in all environments included in the study. The genotypes G5 and G4 present high mean productivity. However, they were unstable and have a specific adaptation to the environments of high quality. The environment E12 gives the highest mean 
productivity (7516.500 kg/ha) and environment E19 has the lowest mean $(4837.614 \mathrm{~kg} / \mathrm{ha})$. These can be considered examples of favourable and unfavourable environments respectively.

Among SVD-based statistical analyses, AMMI is a unique analysis that completely and always separates G, E, and GEI as required for most agricultural research purposes. Furthermore, it separates signal from noise as well as any other method for the purpose of gaining accuracy. AMMI analysis of multi-environment yield trials serves two main purposes: (i) understanding complex GEI, including delineating mega-environments and selecting genotypes to exploit narrow adaptations, and (ii) gaining accuracy to improve recommendations, repeatability, selections, and genetic gains.

\section{Acknowledgements}

The first author thanks the National Council of Technological and Scientific Development (CNPq), Brazil for financial support; the second author thanks the Academy of Sciences for the Developing World (TWAS), Italy (CNPq-TWAS programme) and CNPq for financial support.

\section{REFERENCES}

Annicchiarico P. (1997): Additive main effects and multiplicative interaction (AMMI) analysis of genotype-location interaction in variety trials repeated over years. Theor. Appl. Genet. 94: 1072-1077.

Annicchiarico P. (2002): Genotype $\times$ environment interaction: Challenges and opportunities for plant breeding and cultivar recommendations. Food and Agriculture Organization of the United Nations. FAO, Rome, Italy.

Arciniegas-Alarcn S., Garcia-Peña M., Dias C.T.S., Krzanowski W. J., (2010): An alternative methodology for imputing missing data in trials with genotypeby-environment interaction. Biometrical Letters 47: 1-14.

Cornelius P.L., Crossa J. (1999): Prediction assessment of shrinkage estimators of multiplicative models for multi-environment trials. Crop Science 39: 9981009.

Cornelius P.L., Seyedsar M., Crossa J. (1992): Using the shifted multiplicative model to search for "separability" in crop cultivar trials. Theoretical and Applied Genetics 84: 161-172.

Dias C.T.S., Krzanowski W.J. (2006): Choosing components in the additive main effect and multiplicative interaction (AMMI) models.Scientia Agricola 63: $169-175$.

Dias C.T.S., Krzanowski W.J. (2003): Model selection and cross validation in additive main effect and multiplicative interaction models. Crop Science 43: 865-873. 
Falconer D.S., Mackay T.F.C. (1996): Introduction to quantitative genetics. 4nd ed. Edinburgh: Longman Group Limited.

Gabriel K.R. (1971): The biplot graphic display of matrices with application to principal components analysis. Biometrika 58: 453-467.

García-Peña M., Dias C.T.S. (2009): Analysis of bivariate additive models with multiplicative interaction (AMMI). Biometric Brazilian Journal 27(4): 586602.

Gauch H.G. (1988): Model selection and validation for yield trials with interaction. Biometrics 44(3): 705-715.

Gauch H.G. (1992): Statistical analysis of regional yield trials: AMMI analysis of factorial designs. Elsevier, Amsterdam.

Gauch H.G. (2006): Statistical analysis of yield trials by AMMI and GGE. Crop Science 46: 1488-1500.

Gauch H.G. (2013): A Simple Protocol for AMMI Analysis of Yield Trials. Crop Science:(in press).

Gauch H.G., Zobel R.W. (1988): Predictive and postdictive success of statistical analysis of yield trials. Theoretical and Applied Genetics 76: 1-10.

Gauch H.G., Zobel R.W. (1996): AMMI analysis of yield trials. In Genotype by environment interacrtion, pp. 85-122. Eds Kang M.S., Gauch H.G. New York, USA: CRC Press.

Gauch H.G.; Piepho H.P.; Annicchiarico P. (2008): Statistical analysis of yield trials by AMMI and GGE: Further considerations. Crop Sci. 48: 866-889.

Gauch H.G., Rodrigues P.C., Munkvold J.D., Heffner E.L., Sorrells M. (2011): Two New Strategies for Detecting and Understanding QTL $\times$ Environment Interactions. In Crop Sci 51: 96-113.

Gollob H.F. (1968): A statistical model which combines feature of factor analitic and analysis of variance techniques. Psychometrika 33: 73-115.

Piepho H. P. (1995): Robustness of statistical test for multiplicative terms in the additive main effects and multiplicative interaction model for cultivar trials. Theoretical and Applied Genetics, 90(3/4): 438-443.

Rodrigues P.C., Malosetti M., Gauch H. G., Van Eeuwijk F.A. (2014): A weighted AMMI algorithm to study genotype-by-environment interaction and QTLby-environment interaction. Crop Science.

Smith M.F., Gauch H.G. (1992): Effects of noise on AMMI and hierarchical classification analyses. South African Statist J. 26: 121-142.

SAS Institute. (2004): SAS/IML 9.1 User.s guide. Carey: SAS Institute Inc.

Yan W., Kang M.S., Ma B., Woods S., Cornelius P.L. (2007): GGE biplot vs. AMMI analysis of genotype-by-environment data. Crop Sci. 47: 643-655.

Yang R. C., Crossa J., Cornelius P.L., Burgueño J. (2009): Biplot analysis of genotype $\times$ environment interaction: Proceed with caution. Crop Sci. 49: $1564-1576$.

Yan W. (2010): Optimal Use of Biplots in Analysis of Multi-Location Variety Test Data. Acta Agronomica Sinica, 36 (11): 1805-1819. 